Review

\title{
Immunological Features of the Non-Structural Proteins of Porcine Reproductive and Respiratory Syndrome Virus
}

\author{
Edgar Rascón-Castelo ${ }^{1}$, Alexel Burgara-Estrella ${ }^{1}$, Enric Mateu ${ }^{2,3}$ and Jesús Hernández 1,* \\ 1 Laboratorio de Inmunología, Centro de Investigación en Alimentación y Desarrollo A.C (CIAD) \\ Carretera a la Victoria Km 0.6, C.P. 83304 Hermosillo, Sonora, Mexico; \\ E-Mails: erascon83@gmail.com (E.R.-C.); alexel.burgara@gmail.com (A.B.-E.) \\ 2 Centre de Recerca en Sanitat Animal (CReSA), UAB-IRTA, Campus de la Universitat Autònoma \\ de Barcelona, 08193 Bellaterra, Barcelona, Spain; E-Mail: enric.mateu@uab.cat \\ 3 Departament de Sanitat i d'Anatomia Animals, Universitat Autònoma de Barcelona, \\ 08193 Bellaterra, Barcelona, Spain \\ * Author to whom correspondence should be addressed; E-Mail: jhdez@ciad.mx; \\ Tel./Fax: +52-662-280-0010.
}

Academic Editor: Eric O. Freed

Received: 5 November 2014 / Accepted: 15 February 2015 / Published: 24 February 2015

\begin{abstract}
Porcine reproductive and respiratory syndrome virus (PRRSV) is currently one of the most important viruses affecting the swine industry worldwide. Despite the large number of papers published each year, the participation of non-structural proteins (nsps) in the immune response is not completely clear. nsps have been involved in the host innate immune response, specifically, nsp1 $\alpha / \beta$, nsp2, nsp4 and nsp11 have been associated with the immunomodulation capability of the virus. To date, only participation by nsp1, nsp2, nsp4 and nsp7 in the humoral immune response has been reported, with the role of other nsps being overlooked. Furthermore, nsp1, nsp2, nsp5, nsp7 nsp9, nsp10, nsp11 have been implicated in the induction of IFN- $\gamma$ and probably in the development of the cell-mediated immune response. This review discusses recent reports involving the participation of nsps in the modulation of the innate immune response and their role in the induction of both the humoral and cellular immune responses.
\end{abstract}

Keywords: PRRSV; non-structural proteins; immune response 


\section{Introduction}

Porcine reproductive and respiratory syndrome (PRRS) was first described in the USA in 1987 [1] and appeared in Europe soon thereafter [2]. The causal agent, PRRS virus (PRRSV), was initially identified in Europe [2] and reported one year later in the USA [3]. Since then, PRRSV has become one of the pathogens that has the greatest impact on the swine industry, causing losses in the USA estimated at approximately 664 million US dollars per year [4].

PRRSV is a single-stranded, positive-sense RNA enveloped virus that belongs to the Arteriviridae family, together with equine arteritis virus (EAV), lactate dehydrogenase-elevating virus (LDV) and simian hemorrhagic fever virus (SHFV) [5]. The viral genome is $15 \mathrm{~kb}$ and contains at least 10 open reading frames (ORFs) flanked by two untranslated regions 5' and 3'. ORFs $1 \mathrm{a}$ and $1 \mathrm{~b}$, account for 75\% of the viral genome and encode two long polypeptides (pp), ppla and pplab; after enzymatic cleavage, these pp produce 14 non-structural proteins (nsps) that are implicated in viral replication, and two additional viral proteins called $\mathrm{nsp} 2 \mathrm{TF}$ and nsp2N that results from ribosomal frameshifting [6,7]. The proteolytic process leading to the cleavage of nsps is performed by four viral proteases, encoded in the nsp1 $\alpha, n s p 1 \beta, n s p 2$ and nsp4 regions. ORF2 to ORF7 are located at the 3' terminus and code for eight structural proteins designated as GP2, E, GP3, GP4, GP5, ORF5a protein, M and N (Figure 1) [8-11].

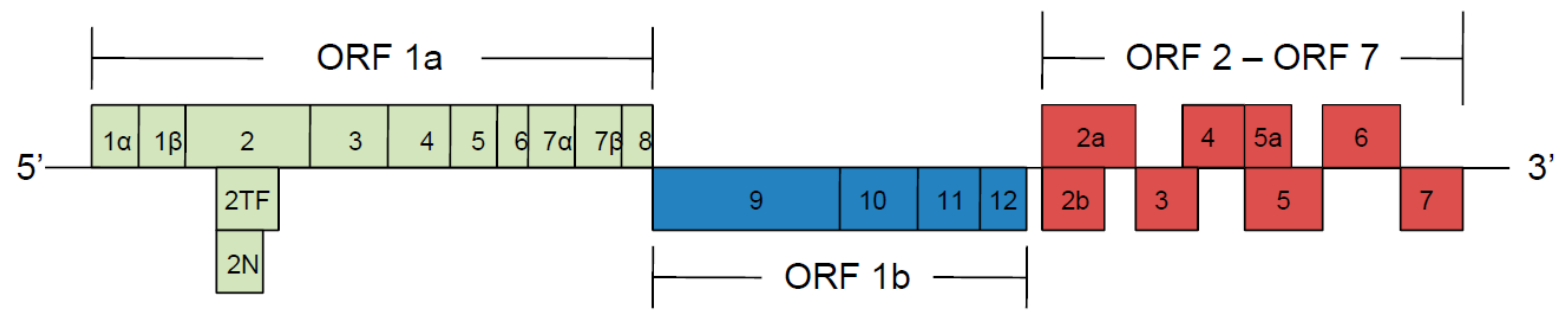

Figure 1. Porcine reproductive and respiratory syndrome virus (PRRSV) genome organization. ORF1a and $1 \mathrm{~b}$ are translated into pp1a and pplab, which after proteolytic processing produce at least 14 nsps. ORF2 to ORF7 encode structural proteins associated with virion infectivity.

Two genotypes of PRRSV have been described thus far: genotype 1, also known as the European type; and genotype 2, the North American type [12]. Both genotype 1 and 2 PRRSV infect cells of the monocyte/macrophage lineage. Homology between the genotypes varies depending on the gene or the protein that is being compared. Overall (for both genotypes), among the non-structural proteins, nsp1 and nsp2 are the most variable parts of the virus, with an identity of $50.5 \%$ to $54.3 \%$ and $24.4 \%$ to $28.0 \%$, respectively; between the genotypes, nsp9 shows the highest conservation, with an identity of $73.2 \%$ to $75.0 \%$. The most conserved structural protein is M protein (75.2\% to $81.6 \%$ identity) [13].

PRRSV nsps are the first proteins to be expressed after infection and perform essential roles in viral replication (nsp9 to nsp12), with some being associated with virulence (nsp3 to nsp8) [14]. Recent studies have revealed the importance of nsps in the modulation of the immune response of pigs infected with PRRSV, but little information is available about the immune response elicited against these proteins. Here, we discuss recent reports involving the role of nsps in the modulation of the innate immune response as an evasion strategy of PRRSV and the possible role of nsps as a target for a protective immune response. 


\section{Non-Structural Proteins (nsps)}

Fang and Snijder (2010) published an excellent review on the biological functions of the non-structural proteins of PRRSV that can be consulted for in-depth information [15]. The following is a brief summary of the roles of PRRSV nsps.

nsp1 is a 383-amino acid, multifunctional protein located at the amino terminus of pp1a and contains two subunits: nsp1 $\alpha$ and nsp1 $\beta$. Although nsp1 $\alpha$ appears to be relatively conserved, $n s p 1 \beta$ is highly variable [13]. Cleavage of nsp1 is autocatalytic and is thought to occur at Met or His 180 (depending on the genotype) [16]. The papain-like protease activity of nsp1 $\alpha$ resides mainly in residues Cys 76 and His 146 or 147, whilst the papain-like protease activity of nsp1 $\beta$ resides in Cys 276 and His 345 [17]. Due to its zinc finger motifs, the participation of nsp1 is essential for the transcription of subgenomic mRNAs that are produced during the replication of arteriviruses, and certain point mutations in this protein can block the replication of PRRSV $[18,19]$. Kroese et al. (2008) mentioned that the loss of the papain-like-protease activity of nspl $\alpha$ is related to the inhibition of sg mRNA synthesis, though the replication of genomic RNA is not affected. In addition, the loss of the papain-like-protease activity of $\mathrm{nsp} 1 \beta$ results in the non-processing of the $\mathrm{nsp} 1 \beta / \mathrm{nsp} 2$ junction in vitro; therefore, no signs of viral RNA synthesis is detected [17].

nsp2 is the most variable of the PRRSV nsps, particularly the central region of nsp2. Several deletions have been reported in both genotype 1 [13,20,21] and genotype 2 strains [22-24], suggesting that nsp2 possesses functions non-essential for replication but that could be important/essential for nsp2's function in host immunity. This protein has four functional regions: an $\mathrm{NH}_{2}$-terminus with a papain-like proteinase domain (PLP2) [25]; a non-specific central functional region, which differs in size and amino acid composition between PRRSV isolates; a hydrophobic region at the C-terminus and a conserved C-terminal tail $[26,27]$. nsp2 is produced in large quantities by infected cells and, together with nsp 3 and nsp5, modifies the membrane of infected cells, favoring the assembly of the multi-protein complex of viral replication. In addition, nsp2 serves as cofactor for the function of nsp4 [28,29]. Recently, it has been shown that nsp2 may be associated with the virion [30], a fact that could explain why antibodies against nsp2 are immunodominant in infected pigs [31,32].

nsp4 is a chymotrypsin-like serine proteinase that is considered to be the most important virus protease since it contains a catalytic triad (His 1103, Asp 1129 and Ser 1184) that cleave nsp3 to nsp12 [33,34]. nsp9 is a highly conserved RNA-dependent RNA polymerase, whereas nsp10 is a helicase that presents a metal-complexing region at its amino terminus [34]. The functions of proteins nsp11 and nsp12 remain unknown, though has been reported that nsp11 shows specific pyrimidine endo-ribonuclease activity as well as a conserved domain present in all nidoviruses [35]. It has been suggested that the nsp3, nsp5, nsp6, nsp7, and nsp8 proteins are responsible for the virulence of PRRSV [14]. Recently, Li et al. found that the nsp9 and nsp10 increase the virulence of the atypical HP-PRRSV emerging in China [36], but information regarding mechanisms by which they could contribute to pathogenicity remain unknown.

\section{Modulation of Innate Immune Responses by Non-Structural Proteins}

Type I interferons are key elements of the innate immune response involved in viral infections. Among their functions include: (a) the generation of an antiviral state in cells that blocks viral 
replication; (b) the promotion of the development of $\mathrm{CD}^{+} \mathrm{T}$ cells; (c) the regulation of MHC-II (major histocompatibility complex); (d) the promotion of the differentiation of antigen-presenting cells; (e) the stimulation of the proliferation of memory cells through IL-15; and (f) the prolongation of the half live of activated T lymphocytes [37,38].

Many viruses [39-41], including PRRSV [42-45], have mechanisms for inhibiting the production of interferons to evade the immune system. PRRSV has been shown to be a poor IFN inducer in in vitro models of macrophages [46]. The inhibition of type I IFNs has been shown to be strain dependent for plasmacytoid dendritic cells, whereas inhibition of IFN- $\alpha$ and IFN- $\beta$ appears to be more general in myeloid DCs, macrophages and non-macrophage cell lines such as MARC-145 and BHK-21 [47,48].

The inhibition of type I IFNs has been largely attributed to the ability of nsps to inhibit the promoter of IFN- $\beta$ by blocking the translocation of IRF3 and the inhibition of NF-kappaB (NF- $\kappa \mathrm{B})$, though other mechanisms are thought to exist. As shown by Calzada-Nova et al. a UV-inactivated virus retains the ability to inhibit IFN- $\alpha$ responses of plasmacytoid DCs, a fact that indicates that inhibition may also be related to the interaction of PRRSV with the surface receptors of plasmacytoid DCs [49]. However, in addition to a decrease in IFN expression, the lack of IFNs affects the expression of other proteins important to anti-viral response, including interferon-stimulated genes (ISGs), such as the protein produced by the gene ISG15 [50]. It has been observed that in contrast to what occurs with the classical genotype 2 North American isolates, neither protein kinase R (PKR), 2',5'-oligoadenylate synthetase (OAS) or MX are induced in highly virulent PRRSV infection, suggesting that the production of IFN is decreased by the down-regulation of ISG [51].

Recent studies have shown the participation of individual PRRSV proteins in IFN inhibition. nsp1 (both subunits $\alpha$ and $\beta$ ) has been reported to be one of the main elements involved in the inhibition of type I IFNs [52-54]. nsp1 has been shown to suppress the activity of NF- $\kappa$ B by inhibiting I $\mathrm{BB} \alpha$ phosphorylation, resulting in the down-regulation of the IFN promoter [55,56]. Interestingly, this ability is strongly mediated by the C-terminus of nsp1 $\alpha$. In fact, the inhibitory potential is lost when this region is deleted [55]. Additionally, nsp1 $\alpha$ degrades CREB-binding protein, preventing the recruitment of IRF3 for enhanceosome assembly and consequently blocking IFN induction [57,58] (Table 1).

Table 1. Inhibition of type I IFNs is the result of multiple nsps and mechanisms*.

\begin{tabular}{|c|c|c|c|}
\hline nsp & Cell Tested & Mechanism & Reference \\
\hline \multirow[t]{3}{*}{ nsp1 } & MARC-145, HEK-293T & $\downarrow$ phosphorylation of IRF3 & {$[52,56]$} \\
\hline & MARC-145, HeLa & Degradation of CREB-binding protein & [57] \\
\hline & $\mathrm{HeLa}$ & $\downarrow$ IFN- $\beta$ promoter activation & [52] \\
\hline \multirow[t]{3}{*}{ nsp1a } & HEK-293, HT1080 & $\downarrow$ IRF3-mediated gene activation & [52] \\
\hline & HEK-293T & $\downarrow$ IFN- $\beta$ promoter activation & [53] \\
\hline & $\mathrm{HeLa}$ & $\downarrow$ IкB phosphorylation and nuclear translocation & [55] \\
\hline \multirow[t]{2}{*}{ nsp1及 } & HEK-293, HT1080 & $\downarrow$ IRF3-mediated gene activation & {$[52]$} \\
\hline & HEK-293T & No effect on IRF3 & [53] \\
\hline \multirow[t]{3}{*}{ nsp2 } & HEK-293T & $\downarrow$ function as a deubiquitinating enzyme & [59] \\
\hline & & $\downarrow$ phosphorylation of IRF3 & {$[60]$} \\
\hline & $\mathrm{HeLa}$ & $\downarrow$ IFN- $\beta$ promoter activation & [52] \\
\hline nsp4 & HeLa, MARC-145 & $\downarrow$ IFN- $\beta$ promoter activation & {$[52,61,62]$} \\
\hline \multirow[t]{2}{*}{ nsp11 } & HEK-293, HT1080, MARC-145 & $\downarrow$ IRF3-mediated gene activation & {$[52,63]$} \\
\hline & HeLa, MARC-145 & $\downarrow$ IFN- $\beta$ promoter activation & {$[52,63]$} \\
\hline
\end{tabular}

* Adapted from [44]. 
The central region of nsp2 has been shown to play an important role in the regulation of the innate immune response, and the protein contains several linear immunodominant B-epitopes designated ES2 to ES7 [32]. Mutant viruses harboring a deletion in the region corresponding to ES3 (located in the central hypervariable region) have an increased cytolytic activity and replicate to higher titers than the parental strain. This situation correlated with a decreased potential for inducing IL- $1 \beta$ and TNF- $\alpha$ release by infected macrophages, an indication that nsp2 is involved in the regulation of the innate response and most likely in attenuation/virulence [64]. Recently, it has been shown that nsp2 indeed does contain different domains with several functions. In HeLa cells, nsp2 may activate the NF- $\kappa$ B pathway; because this was related to the hypervariable region of nsp2 [65], the effect could be different depending on the viral strain. In addition, the N-terminal PLP2 domain of PRRSV nsp2 is capable of inhibiting the ubiquitination of retinoic acid-inducible gene 1-a pattern recognition receptor, which binds double-stranded RNA, interfering with the innate response of the cell $[59,66]$.

Chen et al. found that nsp4 of the highly pathogenic PRRSV (HP-PRRSV) strain JXwn06 has severe inhibitory effects on IFN- $\beta$, NF- $\kappa$ B and IRF3 compared to the low pathogenic PRRSV strain HB-1/3.9. These effects were demonstrated with a change in aminoacids at residue 155 (Thr to Lys); this change improved nuclear localization and inhibition of IFN- $\beta$ transcription by nsp4. This study suggests that the nuclear localization of nsp4 could have an important role in the viral replication of PRRSV, besides its characteristic serine protease activity in the cytoplasm [61,67]. A recent report describes that nsp4 suppress IFN- $\beta$ expression through the interference of NF- $\kappa$ B signaling pathway by targeting the NF- $\mathrm{B}$ essential modulator (NEMO), suggesting that targeting NEMO could be an effective strategy for viruses to inhibit the NF- $\kappa$ B pathway [62].

In EAV, Endoribonuclease (NendoU) activity has three catalytic residues (Hys-126, Hys-146 and Lys-170) [35], in PRRSV, this site is located at residues Hys-129, Hys-144 and Lys-173. In a mutagenesis assays performed by Shi et al. the deletion on Hys-129 disrupts this activity and also loses the ability to block the IFN- $\beta$ promoter [54]. In agreement with these results, Sun et al. demonstrated that the PRRSV nsp11 was able to inhibit the IFN- $\beta$ expression in a dose-dependent way on MARC-145 cells [63].

Information on other nsps involved in the regulation of the innate immune response is scarce. Recently, Han et al. [68] demonstrated that nsp1 subunits of others arteriviruses such as EAV, LDV and SHFV, are able to modulate IFN production by inhibiting IFN promoters or blocking signaling pathways (IRF3 and NF- $\mathrm{KB}$ ). As it is plausible that there may be other regions of PRRSV nsps involved in the positive or negative regulation of the immune response, it is essential to identify the viral proteins and their domains that have the capacity to inhibit innate responses in an effort to understand how the virus may subvert the innate immune response and to develop effective vaccines.

\section{Humoral Immune Response to Non-Structural Proteins}

In the course of PRRSV infection, antibodies develop rapidly, mainly against the N protein; however, these early antibodies are not protective and may also contribute to the phenomenon of antibodydependent enhancement (ADE) [69], though ADE has not been confirmed in the field. Neutralizing antibodies appear later and are thought to be directed principally against GP5, GP4, GP3 and most likely 
GP2 [70-73]. Most studies pursuing antibodies directed at nsps have focused on the potential for differentiating between strains, either for vaccination or for differentiation between genotypes.

Using a phage display system, Oleksiewicz et al. showed that linear B-epitopes could be found in nsp1, nsp2, and nsp4, and the nsp2 epitopes were considered to be immunodominant (Table 2) [32]. Subsequently, de Lima et al. prepared a collection of 97 overlapping synthetic peptides from nsp2 and tested them with sera from PRRSV hyperimmune pigs [31]. Their results showed that 10 of the $97 \mathrm{nsp} 2$ peptides were recognized by more than $80 \%$ of the sera from pigs that had been infected for up to 90 days with the North American-type NVSL 97-7895. The majority of those peptides contained regions rich in hydrophilic amino acids which may enhance the recognition by B-lymphocytes [31], and some of them were located in non-essential regions of nsp2 that could be deleted without affecting the viability of the virus, thus having the potential to be used for differential diagnosis [74]. Unfortunately, several reports indicate that deletions in nsp2 are very frequent in wild-type PRRSV, compromising the use of this protein as a marker for differential vaccines [13,20-22].

Table 2. Role of nsps in the immune response.

\begin{tabular}{|c|c|c|c|}
\hline nsp & Humoral response & Cellular response & Reference \\
\hline \multirow[t]{4}{*}{ nsp 1} & $\sqrt{ }$ & & {$[75]$} \\
\hline & $\sqrt{ }$ & & {$[76]$} \\
\hline & $\sqrt{ }$ & & {$[32]$} \\
\hline & & $\sqrt{ }$ & [77] \\
\hline \multirow[t]{5}{*}{ nsp2 } & $\sqrt{ }$ & & {$[75]$} \\
\hline & $\sqrt{ }$ & & {$[31]$} \\
\hline & $\sqrt{ }$ & & [76] \\
\hline & $\sqrt{ }$ & & [32] \\
\hline & & $\sqrt{ }$ & [77] \\
\hline \multirow[t]{2}{*}{ nsp4 } & $\sqrt{ }$ & & {$[76]$} \\
\hline & $\sqrt{ }$ & & {$[32]$} \\
\hline \multirow[t]{2}{*}{ nsp5 } & & $\sqrt{ }$ & {$[78]$} \\
\hline & & $\sqrt{ }$ & [77] \\
\hline \multirow[t]{2}{*}{ nsp7 } & $\sqrt{ }$ & & [75] \\
\hline & & $\sqrt{ }$ & [77] \\
\hline \multirow[t]{2}{*}{ nsp9 } & & $\sqrt{ }$ & [79] \\
\hline & & $\sqrt{ }$ & {$[77]$} \\
\hline nsp10 & & $\sqrt{ }$ & [79] \\
\hline nsp11 & & $\sqrt{ }$ & {$[78]$} \\
\hline
\end{tabular}

Other authors have described similar results for recombinant nsp1 and nsp2 [76] and showed that recombinant nsp1 and nsp2 could be recognized by sera obtained from pigs infected with different PRRSV strains. Although it was not possible to identify conserved regions, a highly variable region was located, suggesting that this region could be a marker for distinguishing between vaccinated and infected animals.

At the initial stages of infection, the kinetics of the humoral response against nsp2 are similar to that of the $\mathrm{N}$ protein, though in the long term, antibodies against nsp2 persist for longer than anti-N antibodies [80]. Memory B-cells producing anti-nsp antibodies are found mainly in the tonsils during 
the persistent stages of the infection [80]. Brown et al. [75] evaluated the humoral response to nsp1, nsp2 and nsp7 proposing that nsp7 is a good candidate for diagnosis and could be evaluated as a marker for differentiating infections of genotypes 1 and 2 .

The humoral response to other arterivirus nsps is scarce. Go et al. [81] expressed recombinant nsp1 to nsp12 and evaluated the immune response by immuneprecipitation using serum samples of experimental infected horses with different strains $(n=3)$, persistently infected horses $(n=3)$ and vaccinated horses with a modified live vaccine $(n=4)$. Results showed that $n s p 2, n s p 4, n s p 5$ and nsp12 were the most immunogenic nsps of EAV. nsp1, nsp3, nsp6 and nsp11 were not immunoprecipitated by the serum samples. It is interesting to remark that all serum samples contained VN antibodies titers. These data suggest a differential humoral response against EAV nsps as observed in PRRSV nsps.

\section{Cell-Mediated Immune Response to Non-Structural Proteins}

It is generally accepted that PRRSV induces an unusual adaptive immune response, with a delayed development of neutralizing antibodies and the cell-mediated response [82,83], as previously reviewed [84], and this response is thought to be largely caused by the ability of the virus to modulate the immune response. However, it is generally accepted that once the virus is cleared and immunity develops in full, immunity against a homologous re-infection is generally sterilizing, whereas protection against a heterologous strain is only partial.

The participation of nsps in the development of the cell-mediated immune response has received comparatively little attention. A study by Parida et al. describes peptides present in nsp9 and nsp 10 as capable of inducing effector/recall IFN- $\gamma$ responses [79]. In that study, the authors synthesized 17-mer peptides from both proteins (nsp9 and nsp10 of PRRSV genotype 2 strain FL-12) to determine their capacity for inducing the proliferation of $T$ cells. Initially, they identified groups of reactive peptides that were then tested individually; those that retained their capacity for stimulating proliferation were tested to determine their capacity to induce the production of IFN- $\gamma$. Finally, four peptides from each protein were identified as having the capacity to induce both a proliferative response and a response for IFN- $\gamma$; three of the four peptides were found in conserved regions of the proteins. In another study that used bioinformatics to predict T-cell epitopes from conserved regions of PRRSV genotype 1 and 2 followed by in vitro confirmation, the authors described peptides from nsp 2 and nsp 5 capable of inducing IFN- $\gamma$ responses [78]. Recent results from our laboratory also indicate that certain peptides from nsp2, nsp5, nsp9, and nsp11 are able to induce IL-10 [78] in recall responses. As described by Burgara-Estrella et al. [78] some of the IL-10-inducing peptides were able to inhibit the production of IFN- $\gamma$ induced by PHA [78]. These results suggest that the capacity to modulate the immune response through IL-10 is an intrinsic property of some nsps of PRRSV. This redundancy in induction represents a complicated challenge that remains to be resolved (Table 2).

Recently, a study carried out by Mokhtar et al. [77] reported a synthetic overlapping peptide library representing the 19 proteins of PRRSV genotype 1 composed of 127615 -mer peptides. The aim of that study was to elucidate the specificity of the T cell response induced by PRRSV 1 infection, as well as to determine the phenotype of the $\mathrm{T}$ cells responding to a given peptide. Mokhtar et al. [77] found T cells that produced IFN- $\gamma$ when stimulated with peptides from nsp1, nsp2, nsp7, nsp9 and nsp11 as well as other peptides from N, M and GP3 structural proteins. The above response was observed in two 
of three pigs repeatedly infected with PRRSV; despite the limited number of pigs, the data indicate a marked diversity in the response to nsps, which is most likely attributable to the MHC haplotype. However, the results also demonstrate that the conserved regions in nsps (such as nsp1 $\beta_{149-163)}$ can be highly antigenic. In this same study, the authors tested two additional groups of pigs infected with two different PRRSV strains. Those pigs that were infected with PRRSV strains with closely related peptide sequences showed a significant response to $n s p 1 \beta$ and nsp2 peptides, and this response was similar to that in pigs infected with divergent PRRSV strains [77]. Therefore, the similarities of responses among divergent strains can be attributed to the rate of conservation of the peptides between divergent strains. It is important to mention that Mokhtar et al. and Parida et al. used overlapping peptides, in contrast, Burgara-Estrella et al. used bioinformatics tools to predict T-cell epitopes. Despite this discrepancy, the above studies revealed the participation of nsps in the induction of IFN- $\gamma$. Further studies will reveal which nsps peptides or nsps are able to induce protective immunity against PRRSV.

\section{Conclusions}

Due to the importance of PRRSV in the swine industry, much information about the mechanisms of regulation has been reported. Although the current knowledge of these mechanisms is insufficient to completely understand the immuno-pathogenesis of the virus, recent studies have focused on the non-structural proteins involved in modulating and inducing the immune response. Scarce information about nsps regarding the cellular response has been reported; however, studies demonstrate that nsps contain regions that are highly antigenic and conserved between different PRRSV strains, suggesting that the utilization of some nsps as a target of the immune response is more important than initially thought. In the future, vaccines based on conserved immunogenic nsps could induce protection against different strains, with an effective and rapid response to infection and preventing the immuno-modulatory effects of the virus. Within this scenario, nsps could be good candidates for in-depth exploration.

\section{Conflicts of Interest}

The authors declare that they have no competing interests.

\section{References}

1. Keffaber, K.K. Reproductive failure of unknown etiology. Am. Assoc. Swine Pract. Newsl. 1989, 1, $1-9$.

2. Wensvoort, G.; Terpstra, C.; Pol, J.M.; ter Laak, E.A.; Bloemraad, M.; de Kluyver, E.P.; Kragten, C.; van Buiten, L.; den Besten, A.; Wagenaar, F.; et al. Mystery swine disease in The Netherlands: The isolation of Lelystad virus. Vet. Q. 1991, 13, 121-130.

3. Collins, J.E.; Benfield, D.A.; Christianson, W.T.; Harris, L.; Hennings, J.C.; Shaw, D.P.; Goyal, S.M.; McCullough, S.; Morrison, R.B.; Joo, H.S.; et al. Isolation of swine infertility and respiratory syndrome virus (isolate ATCC VR-2332) in North America and experimental reproduction of the disease in gnotobiotic pigs. J. Vet. Diagn. Investig. 1992, 4, 117-126. 
4. Holtkamp, D.J.; Kliebenstein, J.B.; Neumann, E.J.; Zimmerman, J.; Rotto, H.F.; Yoder, T.K.; Wang, C.; Yeske, P.; Mowrer, C.L.; Haley, C.A. Assessment of the economic impact of porcine reproductive and respiratory syndrome virus on United States pork producers. J. Swine Health. Prod. 2013, 21, 72-84.

5. Cavanagh, D. Nidovirales: A new order comprising Coronaviridae and Arteriviridae. Arch. Virol. 1997, 142, 629-633.

6. Li, Y.; Treffers, E.E.; Napthine, S.; Tas, A.; Zhu, L.; Sun, Z.; Bell, S.; Mark, B.L.; van Veelen, P.A.; van Hemert, M.J.; et al. Transactivation of programmed ribosomal frameshifting by a viral protein. Proc. Natl. Acad. Sci. USA 2014, 111, E2172-E2181.

7. Fang, Y.; Treffers, E.E.; Li, Y.; Tas, A.; Sun, Z.; van der Meer, Y.; de Ru, A.H.; van Veelen, P.A.; Atkins, J.F.; Snijder, E.J.; et al. Efficient -2 frameshifting by mammalian ribosomes to synthesize an additional arterivirus protein. Proc. Natl. Acad. Sci. USA 2012, 109, E2920-E2928.

8. Allende, R.; Lewis, T.L.; Lu, Z.; Rock, D.L.; Kutish, G.F.; Ali, A.; Doster, A.R.; Osorio, F.A. North American and European porcine reproductive and respiratory syndrome viruses differ in non-structural protein coding regions. J. Gen. Virol. 1999, 80, 307-315.

9. Firth, A.E.; Zevenhoven-Dobbe, J.C.; Wills, N.M.; Go, Y.Y.; Balasuriya, U.B.; Atkins, J.F.; Snijder, E.J.; Posthuma, C.C. Discovery of a small arterivirus gene that overlaps the GP5 coding sequence and is important for virus production. J. Gen. Virol. 2011, 92, 1097-1106.

10. Johnson, C.R.; Griggs, T.F.; Gnanandarajah, J.; Murtaugh, M.P. Novel structural protein in porcine reproductive and respiratory syndrome virus encoded by an alternative ORF5 present in all arteriviruses. J. Gen. Virol. 2011, 92, 1107-1116.

11. van Dinten, L.C.; Rensen, S.; Gorbalenya, A.E.; Snijder, E.J. Proteolytic processing of the open reading frame $1 b$-encoded part of arterivirus replicase is mediated by nsp4 serine protease and is essential for virus replication. J. Virol. 1999, 73, 2027-2037.

12. Meng, X.J.; Paul, P.S.; Halbur, P.G.; Lum, M.A. Phylogenetic analyses of the putative M (ORF 6) and $\mathrm{N}$ (ORF 7) genes of porcine reproductive and respiratory syndrome virus (PRRSV): implication for the existence of two genotypes of PRRSV in the U.S.A. and Europe. Arch. Virol. 1995, 140, 745-755.

13. Darwich, L.; Gimeno, M.; Sibila, M.; Diaz, I.; de la Torre, E.; Dotti, S.; Kuzemtseva, L.; Martin, M.; Pujols, J.; Mateu, E. Genetic and immunobiological diversities of porcine reproductive and respiratory syndrome genotype I strains. Vet. Microbiol. 2011, 150, 49-62.

14. Kwon, B.; Ansari, I.H.; Pattnaik, A.K.; Osorio, F.A. Identification of virulence determinants of porcine reproductive and respiratory syndrome virus through construction of chimeric clones. Virology 2008, 380, 371-378.

15. Fang, Y.; Snijder, E.J. The PRRSV replicase: exploring the multifunctionality of an intriguing set of nonstructural proteins. Virus Res. 2010, 154, 61-76.

16. Sun, Y.; Xue, F.; Guo, Y.; Ma, M.; Hao, N.; Zhang, X.C.; Lou, Z.; Li, X.; Rao, Z. Crystal structure of porcine reproductive and respiratory syndrome virus leader protease nsplalpha. J. Virol. 2009, 83, 10931-10940. 
17. Kroese, M.V.; Zevenhoven-Dobbe, J.C.; Bos-de Ruijter, J.N.; Peeters, B.P.; Meulenberg, J.J.; Cornelissen, L.A.; Snijder, E.J. The nsplalpha and nsp1 papain-like autoproteinases are essential for porcine reproductive and respiratory syndrome virus RNA synthesis. J. Gen. Virol. 2008, 89, 494-499.

18. Snijder, E.J.; Wassenaar, A.L.; Spaan, W.J. The 5' end of the equine arteritis virus replicase gene encodes a papainlike cysteine protease. J. Virol. 1992, 66, 7040-7048.

19. Tijms, M.A.; van Dinten, L.C.; Gorbalenya, A.E.; Snijder, E.J. A zinc finger-containing papain-like protease couples subgenomic mRNA synthesis to genome translation in a positive-stranded RNA virus. Proc. Natl. Acad. Sci. USA 2001, 98, 1889-1894.

20. Fang, Y.; Schneider, P.; Zhang, W.P.; Faaberg, K.S.; Nelson, E.A.; Rowland, R.R. Diversity and evolution of a newly emerged North American type 1 porcine arterivirus: Analysis of isolates collected between 1999 and 2004. Arch. Virol. 2007, 152, 1009-1017.

21. Ropp, S.L.; Wees, C.E.; Fang, Y.; Nelson, E.A.; Rossow, K.D.; Bien, M.; Arndt, B.; Preszler, S.; Steen, P.; Christopher-Hennings, J.; et al. Characterization of emerging European-like porcine reproductive and respiratory syndrome virus isolates in the United States. J. Virol. 2004, 78, 3684-3703.

22. Yang, Z.Z.; Fang, W.H.; Habib, M. First results of detection of PRRSV and CSFV RNA by SYBR Green I-based quantitative PCR. J. Vet. Med. 2006, 53, 461-467.

23. Tian, K.; Yu, X.; Zhao, T.; Feng, Y.; Cao, Z.; Wang, C.; Hu, Y.; Chen, X.; Hu, D.; Tian, X.; et al. Emergence of fatal PRRSV variants: unparalleled outbreaks of atypical PRRS in China and molecular dissection of the unique hallmark. PLoS One 2007, 2, e526.

24. Zhou, Y.J.; Hao, X.F.; Tian, Z.J.; Tong, G.Z.; Yoo, D.; An, T.Q.; Zhou, T.; Li, G.X.; Qiu, H.J.; Wei, T.C.; et al. Highly virulent porcine reproductive and respiratory syndrome virus emerged in China. Transbound. Emerg. Dis. 2008, 55, 152-164.

25. Snijder, E.J.; Kikkert, M.; Fang, Y. Arterivirus molecular biology and pathogenesis. J. Gen. Virol.2013, 94, 2141-2163.

26. Fang, L.; Jiang, Y.; Xiao, S.; Niu, C.; Zhang, H.; Chen, H. Enhanced immunogenicity of the modified GP5 of porcine reproductive and respiratory syndrome virus. Virus Genes 2006, 32, 5-11.

27. Ziebuhr, J.; Snijder, E.J.; Gorbalenya, A.E. Virus-encoded proteinases and proteolytic processing in the nidovirales. J. Gen. Virol. 2000, 81, 853-879.

28. Pedersen, K.W.; van der Meer, Y.; Roos, N.; Snijder, E.J. Open reading frame 1a-encoded subunits of the arterivirus replicase induce endoplasmic reticulum-derived double-membrane vesicles which carry the viral replication complex. J. Virol. 1999, 73, 2016-2026.

29. Snijder, E.J.; van Tol, H.; Roos, N.; Pedersen, K.W. Non-structural proteins 2 and 3 interact to modify host cell membranes during the formation of the arterivirus replication complex. J. Gen. Virol. 2001, 82, 985-994.

30. Kappes, M.A.; Miller, C.L.; Faaberg, K.S. Highly divergent strains of porcine reproductive and respiratory syndrome virus incorporate multiple isoforms of nonstructural protein 2 into virions. J. Virol. 2013, 87, 13456-13465.

31. De Lima, M.; Pattnaik, A.K.; Flores, E.F.; Osorio, F.A. Serologic marker candidates identified among B-cell linear epitopes of nsp2 and structural proteins of a north american strain of porcine reproductive and respiratory syndrome virus. Virology 2006, 353, 410-421. 
32. Oleksiewicz, M.B.; Botner, A.; Toft, P.; Normann, P.; Storgaard, T. Epitope mapping porcine reproductive and respiratory syndrome virus by phage display: The nsp2 fragment of the replicase polyprotein contains a cluster of B-cell epitopes. J. Virol. 2001, 75, 3277-3290.

33. van Dinten, L.C.; Wassenaar, A.L.; Gorbalenya, A.E.; Spaan, W.J.; Snijder, E.J. Processing of the equine arteritis virus replicase ORF1b protein: identification of cleavage products containing the putative viral polymerase and helicase domains. J. Virol. 1996, 70, 6625-6633.

34. Wassenaar, A.L.; Spaan, W.J.; Gorbalenya, A.E.; Snijder, E.J. Alternative proteolytic processing of the arterivirus replicase ORF1a polyprotein: evidence that nsp2 acts as a cofactor for the nsp4 serine protease. J. Virol. 1997, 71, 9313-9322.

35. Nedialkova, D.D.; Ulferts, R.; van den Born, E.; Lauber, C.; Gorbalenya, A.E.; Ziebuhr, J.; Snijder, E.J. Biochemical characterization of arterivirus nonstructural protein 11 reveals the nidovirus-wide conservation of a replicative endoribonuclease. J. Virol. 2009, 83, 5671-5682.

36. Li, Y.; Zhou, L.; Zhang, J.; Ge, X.; Zhou, R.; Zheng, H.; Geng, G.; Guo, X.; Yang, H. Nsp9 and nsp10 contribute to the fatal virulence of highly pathogenic porcine reproductive and respiratory syndrome virus emerging in China. PLOS Pathog. 2014, 10, e1004216.

37. Lee, S.M.; Schommer, S.K.; Kleiboeker, S.B. Porcine reproductive and respiratory syndrome virus field isolates differ in in vitro interferon phenotypes. Vet. Immunol. Immunopathol. 2004, 102, 217-231.

38. Tough, D.F. Modulation of T-cell function by type I interferon. Immunol. Cell Biol. 2012, 90, 492-497.

39. Best, S.M.; Morris, K.L.; Shannon, J.G.; Robertson, S.J.; Mitzel, D.N.; Park, G.S.; Boer, E.; Wolfinbarger, J.B.; Bloom, M.E. Inhibition of interferon-stimulated JAK-STAT signaling by a tick-borne flavivirus and identification of NS5 as an interferon antagonist. J. Virol. 2005, 79, 12828-12839.

40. Fernandez-Sesma, A.; Marukian, S.; Ebersole, B.J.; Kaminski, D.; Park, M.S.; Yuen, T.; Sealfon, S.C.; Garcia-Sastre, A.; Moran, T.M. Influenza virus evades innate and adaptive immunity via the NS1 protein. J. Virol. 2006, 80, 6295-6304.

41. Yuan, S.; Wei, Z. Construction of infectious cDNA clones of PRRSV: Separation of coding regions for nonstructural and structural proteins. Sci. China 2008, 51, 271-279.

42. Zhou, L.; Zhang, J.; Zeng, J.; Yin, S.; Li, Y.; Zheng, L.; Guo, X.; Ge, X.; Yang, H. The 30-amino-acid deletion in the nsp2 of highly pathogenic porcine reproductive and respiratory syndrome virus emerging in China is not related to its virulence. J. Virol. 2009, 83, 5156-5167.

43. Yoo, D.; Song, C.; Sun, Y.; Du, Y.; Kim, O.; Liu, H.-C. Modulation of host cell responses and evasion strategies for porcine reproductive and respiratory syndrome virus. Virus Res. 2010, 154, 48-60.

44. Chand, R.J.; Trible, B.R.; Rowland, R.R. Pathogenesis of porcine reproductive and respiratory syndrome virus. Curr. Opin. Virol. 2012, 2, 256-263.

45. Sun, Y.; Han, M.; Kim, C.; Calvert, J.G.; Yoo, D. Interplay between interferon-mediated innate immunity and porcine reproductive and respiratory syndrome virus. Viruses 2012, 4, 424-446.

46. Albina, E.; Carrat, C.; Charley, B. Interferon-alpha response to swine arterivirus (POAV), the porcine reproductive and respiratory syndrome virus. J. Interferon Cytokine Res. 1998, 18, 485-490.

47. Miller, L.C.; Laegreid, W.W.; Bono, J.L.; Chitko-McKown, C.G.; Fox, J.M. Interferon type I response in porcine reproductive and respiratory syndrome virus-infected MARC-145 cells. Arch. Virol. 2004, 149, 2453-2463. 
48. Zhang, C.; Xue, C.; Li, Y.; Kong, Q.; Ren, X.; Li, X.; Shu, D.; Bi, Y.; Cao, Y. Profiling of cellular proteins in porcine reproductive and respiratory syndrome virus virions by proteomics analysis. Virology J. 2010, 7, e242, doi:10.1186/1743-422X-7-242.

49. Calzada-Nova, G.; Schnitzlein, W.M.; Husmann, R.J.; Zuckermann, F.A. North American porcine reproductive and respiratory syndrome viruses inhibit type I interferon production by plasmacytoid dendritic cells. J. Virol. 2011, 85, 2703-2713.

50. Sun, Z.; Li, Y.; Ransburgh, R.; Snijder, E.J.; Fang, Y. Nonstructural protein 2 of porcine reproductive and respiratory syndrome virus inhibits the antiviral function of interferon-stimulated gene 15. J. Virol. 2012, 86, 3839-3850.

51. Xiao, S.; Mo, D.; Wang, Q.; Jia, J.; Qin, L.; Yu, X.; Niu, Y.; Zhao, X.; Liu, X.; Chen, Y. Aberrant host immune response induced by highly virulent PRRSV identified by digital gene expression tag profiling. BMC Genomics 2010, 11, e544.

52. Beura, L.K.; Sarkar, S.N.; Kwon, B.; Subramaniam, S.; Jones, C.; Pattnaik, A.K.; Osorio, F.A. Porcine reproductive and respiratory syndrome virus nonstructural protein 1beta modulates host innate immune response by antagonizing IRF3 activation. J. Virol. 2010, 84, 1574-1584.

53. Chen, Z.; Lawson, S.; Sun, Z.; Zhou, X.; Guan, X.; Christopher-Hennings, J.; Nelson, E.A.; Fang, Y. Identification of two auto-cleavage products of nonstructural protein 1 (nsp1) in porcine reproductive and respiratory syndrome virus infected cells: nsp1 function as interferon antagonist. Virology 2010, 398, 87-97.

54. Shi, X.; Wang, L.; Li, X.; Zhang, G.; Guo, J.; Zhao, D.; Chai, S.; Deng, R. Endoribonuclease activities of porcine reproductive and respiratory syndrome virus nsp 11 was essential for nsp 11 to inhibit IFN-beta induction. Molecular Immunol. 2011, 48, 1568-1572.

55. Song, C.; Krell, P.; Yoo, D. Nonstructural protein 1alpha subunit-based inhibition of NF-kappaB activation and suppression of interferon-beta production by porcine reproductive and respiratory syndrome virus. Virology 2010, 407, 268-280.

56. Shi, X.; Wang, L.; Zhi, Y.; Xing, G.; Zhao, D.; Deng, R.; Zhang, G. Porcine reproductive and respiratory syndrome virus (PRRSV) could be sensed by professional beta interferon-producing system and had mechanisms to inhibit this action in MARC-145 cells. Virus Res. 2010, 153, 151-156.

57. Kim, O.; Sun, Y.; Lai, F.W.; Song, C.; Yoo, D. Modulation of type I interferon induction by porcine reproductive and respiratory syndrome virus and degradation of CREB-binding protein by non-structural protein 1 in MARC-145 and HELA cells. Virology 2010, 402, 315-326.

58. Han, M.; Du, Y.; Song, C.; Yoo, D. Degradation of CREB-binding protein and modulation of type I interferon induction by the zinc finger motif of the porcine reproductive and respiratory syndrome virus nsp1alpha subunit. Virus Res. 2013, 172, 54-65.

59. Sun, Z.; Chen, Z.; Lawson, S.R.; Fang, Y. The cysteine protease domain of porcine reproductive and respiratory syndrome virus nonstructural protein 2 possesses deubiquitinating and interferon antagonism functions. J. Virol. 2010, 84, 7832-7846.

60. Li, H.; Zheng, Z.; Zhou, P.; Zhang, B.; Shi, Z.; Hu, Q.; Wang, H. The cysteine protease domain of porcine reproductive and respiratory syndrome virus non-structural protein 2 antagonizes interferon regulatory factor 3 activation. J. Gen. Virol. 2010, 91, 2947-2958. 
61. Chen, Z.; Li, M.; He, Q.; Du, J.; Zhou, L.; Ge, X.; Guo, X.; Yang, H. The amino acid at residue 155 in nonstructural protein 4 of porcine reproductive and respiratory syndrome virus contributes to its inhibitory effect for interferon-beta transcription in vitro. Virus Res. 2014, 189, 226-234.

62. Huang, C.; Zhang, Q.; Guo, X.K.; Yu, Z.B.; Xu, A.T.; Tang, J.; Feng, W.H. Porcine reproductive and respiratory syndrome virus nonstructural protein 4 antagonizes beta interferon expression by targeting the NF-kappaB essential modulator. J. Virol. 2014, 88, 10934-10945.

63. Sun, Y.; Li, D.; Giri, S.; Prasanth, S.G.; Yoo, D. Differential host cell gene expression and regulation of cell cycle progression by nonstructural protein 11 of porcine reproductive and respiratory syndrome virus. BioMed Res. Int. 2014, 2014, 430508.

64. Chen, Z.; Zhou, X.; Lunney, J.K.; Lawson, S.; Sun, Z.; Brown, E.; Christopher-Hennings, J.; Knudsen, D.; Nelson, E.; Fang, Y. Immunodominant epitopes in nsp2 of porcine reproductive and respiratory syndrome virus are dispensable for replication, but play an important role in modulation of the host immune response. J. Gen. Virol. 2010, 91, 1047-1057.

65. Fang, Y.; Fang, L.; Wang, Y.; Lei, Y.; Luo, R.; Wang, D.; Chen, H.; Xiao, S. Porcine reproductive and respiratory syndrome virus nonstructural protein 2 contributes to NF-kappaB activation. Virology J. 2012, 9, e83.

66. Van Kasteren, P.B.; Beugeling, C.; Ninaber, D.K.; Frias-Staheli, N.; van Boheemen, S.; Garcia-Sastre, A.; Snijder, E.J.; Kikkert, M. Arterivirus and nairovirus ovarian tumor domain-containing deubiquitinases target activated RIG-I to control innate immune signaling. $J$. Virol. 2012, 86, 773-785.

67. Ma, Z.; Wang, Y.; Zhao, H.; Xu, A.T.; Wang, Y.; Tang, J.; Feng, W.H. Porcine reproductive and respiratory syndrome virus nonstructural protein 4 induces apoptosis dependent on its $3 \mathrm{C}$-like serine protease activity. PLOS ONE 2013, 8, e69387.

68. Han, M.; Kim, C.Y.; Rowland, R.R.; Fang, Y.; Kim, D.; Yoo, D. Biogenesis of non-structural protein 1 (nsp1) and nsp1-mediated type I interferon modulation in arteriviruses. Virology 2014, 458-459, 136-150.

69. Cancel-Tirado, S.M.; Yoon, K.J. Antibody-dependent enhancement of virus infection and disease. Viral Immunol. 2003, 16, 69-86.

70. Batista, L.; Pijoan, C.; Dee, S.; Olin, M.; Molitor, T.; Joo, H.S.; Xiao, Z.; Murtaugh, M. Virological and immunological responses to porcine reproductive and respiratory syndrome virus in a large population of gilts. Can. J. Vet. Res. 2004, 68, 267-273.

71. Gonin, P.; Pirzadeh, B.; Gagnon, C.A.; Dea, S. Seroneutralization of porcine reproductive and respiratory syndrome virus correlates with antibody response to the GP5 major envelope glycoprotein. J. Vet. Diagn. Investig. 1999, 11, 20-26.

72. Kwang, J.; Zuckermann, F.; Ross, G.; Yang, S.; Osorio, F.; Liu, W.; Low, S. Antibody and cellular immune responses of swine following immunisation with plasmid DNA encoding the PRRS virus ORF's 4, 5, 6 and 7. Res. Vet. Sci. 1999, 67, 199-201.

73. Weiland, E.; Wieczorek-Krohmer, M.; Kohl, D.; Conzelmann, K.K.; Weiland, F. Monoclonal antibodies to the GP5 of porcine reproductive and respiratory syndrome virus are more effective in virus neutralization than monoclonal antibodies to the GP4. Vet. Microbiol. 1999, 66, 171-186. 
74. de Lima, M.; Kwon, B.; Ansari, I.H.; Pattnaik, A.K.; Flores, E.F.; Osorio, F.A. Development of a porcine reproductive and respiratory syndrome virus differentiable (DIVA) strain through deletion of specific immunodominant epitopes. Vaccine 2008, 26, 3594-3600.

75. Brown, E.; Lawson, S.; Welbon, C.; Gnanandarajah, J.; Li, J.; Murtaugh, M.P.; Nelson, E.A.; Molina, R.M.; Zimmerman, J.J.; Rowland, R.R.; et al. Antibody response to porcine reproductive and respiratory syndrome virus (PRRSV) nonstructural proteins and implications for diagnostic detection and differentiation of PRRSV types I and II. Clin. Vaccine Immunol. 2009, 16, 628-635.

76. Johnson, C.R.; Yu, W.; Murtaugh, M.P. Cross-reactive antibody responses to nsp1 and nsp2 of porcine reproductive and respiratory syndrome virus. J. Gen. Virol. 2007, 88, 1184-1195.

77. Mokhtar, H.; Eck, M.; Morgan, S.B.; Essler, S.E.; Frossard, J.P.; Ruggli, N.; Graham, S.P. Proteome-wide screening of the European porcine reproductive and respiratory syndrome virus reveals a broad range of $\mathrm{T}$ cell antigen reactivity. Vaccine 2014, 32, 6828-6837.

78. Burgara-Estrella, A.; Diaz, I.; Rodriguez-Gomez, I.M.; Essler, S.E.; Hernandez, J.; Mateu, E. Predicted peptides from non-structural proteins of porcine reproductive and respiratory syndrome virus are able to induce IFN-gamma and IL-10. Viruses 2013, 5, 663-677.

79. Parida, R.; Choi, I.S.; Peterson, D.A.; Pattnaik, A.K.; Laegreid, W.; Zuckermann, F.A.; Osorio, F.A. Location of T-cell epitopes in nonstructural proteins 9 and 10 of type-II porcine reproductive and respiratory syndrome virus. Virus Res. 2012, 169, 13-21.

80. Mulupuri, P.; Zimmerman, J.J.; Hermann, J.; Johnson, C.R.; Cano, J.P.; Yu, W.; Dee, S.A.; Murtaugh, M.P. Antigen-specific B-cell responses to porcine reproductive and respiratory syndrome virus infection. J. Virol. 2008, 82, 358-370.

81. Go, Y.Y.; Snijder, E.J.; Timoney, P.J.; Balasuriya, U.B. Characterization of equine humoral antibody response to the nonstructural proteins of equine arteritis virus. Clin. Vaccine Immunol. 2011, 18, 268-279.

82. Meier, W.A.; Galeota, J.; Osorio, F.A.; Husmann, R.J.; Schnitzlein, W.M.; Zuckermann, F.A. Gradual development of the interferon-gamma response of swine to porcine reproductive and respiratory syndrome virus infection or vaccination. Virology 2003, 309, 18-31.

83. Nelson, E.A.; Christopher-Hennings, J.; Benfield, D.A. Serum immune responses to the proteins of porcine reproductive and respiratory syndrome (PRRS) virus. J. Vet. Diagn. Investig. 1994, 6, 410-415.

84. Darwich, L.; Díaz, I.; Mateu, E. Certainties, doubts and hypotheses in porcine reproductive and respiratory syndrome virus immunobiology. Virus Res. 2010, 154, 123-132.

(C) 2015 by the authors; licensee MDPI, Basel, Switzerland. This article is an open access article distributed under the terms and conditions of the Creative Commons Attribution license (http://creativecommons.org/licenses/by/4.0/). 